\title{
FROBENIUS MORPHISMS OF NONCOMMUTATIVE BLOWUPS
}

\author{
TAKEHIKO YASUDA
}

\begin{abstract}
We define the Frobenius morphism of certain class of noncommutative blowups in positive characteristic. Thanks to a nice property of the class, the defined morphism is always flat. Therefore we say that the noncommutative blowups in this class are Kunz regular. One of such blowups is the one associated to a regular Galois alteration. From de Jong's theorem, we see that for every variety over an algebraically closed field of positive characteristic, there exists a noncommutative blowup which is Kunz regular. We also see that a variety with F-pure and FFRT (finite F-representation type) singularities has a Kunz regular noncommutative blowup which is associated to an iteration of the Frobenius morphism of the variety.
\end{abstract}

\section{INTRODUCTION}

The Frobenius morphism is arguably the most important notion in the algebraic geometry of positive characteristic and used almost everywhere. Concerning the singularity theory, Kunz's theorem is classical [11]: A scheme is regular if and only if its Frobenius morphism is flat. The main aim of this article is to define the Frobenius morphism of certain class of noncommutative blowups in positive characteristic and to see that the defined morphism is flat. Here the noncommutative blowup that we mean is basically the same as the noncommutative crepant resolution in [16] and the noncommutative desingularization in [2] except that we remove some assumptions, especially the finiteness of global dimension.

Let $k$ be a field of characteristic $p>0$. Recently it was found in [15 that if $X=\operatorname{Spec} R$ is from some classes of singularities over $k$, then for sufficiently large $e$, the endomorphism $\operatorname{ring} \operatorname{End}_{R}\left(R^{1 / p^{e}}\right)$, whose elements are differential operators on $R^{1 / p^{e}}$, has finite global dimension and is regarded as a noncommutative resolution of $X$. This article derives from the author's attempt to know where the regularity of $\operatorname{End}_{R}\left(R^{1 / p^{e}}\right)$ comes from and to show its regularity for a broader class of singularities. However the regularity which we will consider in this article is the flatness of Frobenius rather than the finiteness of global dimension. It is because the former seems to the author simpler and compatible with $\operatorname{End}_{R}\left(R^{1 / p^{e}}\right)$. We however consider not only noncommutative blowups of the form $\operatorname{End}_{R}\left(R^{1 / p^{e}}\right)$.

Let $X, Y$ be integral normal Noetherian schemes over $k$ with finite Frobenius morphisms and $f: Y \rightarrow X$ a finite dominant morphism. We associate to $f$ a noncommutative blowup $\operatorname{NCB}(Y / X)$, which is the pair of the endomorphism ring $\mathcal{E} n d_{\mathcal{O}_{X}}\left(f_{*} \mathcal{O}_{Y}\right)$ and the left $\mathcal{E} n d_{\mathcal{O}_{X}}\left(f_{*} \mathcal{O}_{Y}\right)$-module $\mathcal{O}_{Y}$. Also we regard this as the category of left $\mathcal{E} n d_{\mathcal{O}_{X}}\left(f_{*} \mathcal{O}_{Y}\right)$-modules with the distinguished object $\mathcal{O}_{Y}$. Let $Y_{e} \rightarrow Y$ be the $e$-times iteration of the Frobenius morphism. We say that $f$ is $F$-steady if for every $e \geq 0$, the structure sheaves of $Y_{e}$ and $Y$ locally have, as $\mathcal{O}_{X}$-modules, the same summands (for details, see Section 4). For instance, if $Y$ is regular, then $f$ is F-steady. Given an F-steady morphism $Y \rightarrow X$, we define the 
Frobenius morphism of $\operatorname{NCB}(Y / X)$, which is flat by construction. Hence we say that $\operatorname{NCB}(Y / X)$ is Kunz regular.

If $k$ is algebraically closed, from de Jong's theorem [4, every $k$-variety $X$ admits a Galois alteration $Y \rightarrow X$ with $Y$ regular. It uniquely factors as $Y \rightarrow \bar{Y} \rightarrow X$ such that $\bar{Y}$ is a normal variety, $Y \rightarrow \bar{Y}$ is finite and $\bar{Y} \rightarrow X$ is a modification. Then the associated noncommutative blowup $\operatorname{NCB}(Y / X)=\operatorname{NCB}(Y / \bar{Y})$ is $\operatorname{Kunz}$ regular. Thus every variety admits a noncommutative blowup which is Kunz regular (Corollary 4.6).

Another interesting example of noncommutative blowups is the one associated to an iterated Frobenius morphism $X_{e} \rightarrow X$ of a normal scheme $X$. In the affine case, this corresponds to the above-mentioned ring $\operatorname{End}_{R}\left(R^{1 / p^{e}}\right)$. If $X$ has only F-pure and FFRT (finite F-representation type) singularities, then for sufficiently large $e, X_{e} \rightarrow X$ is F-steady and the associated noncommutative blowup $\operatorname{NCB}\left(X_{e} / X\right)$ is Kunz regular (see Section [5). The FFRT singularity was introduced in [13] and proved to have $D$-module theoretic nice properties [13, 14. Our result is yet another such property.

1.1. Convention. Throughout the paper, we work over a fixed base field $k$ unless otherwise noted. We mean by a scheme a separated Noetherian scheme over $k$. In Sections 4, 5and 6, we additionally assume that $k$ has characteristic $p>0$ and that every scheme is F-finite, that is, the Frobenius morphism is finite. If $f: Y \rightarrow X$ is an affine morphism of schemes and $\mathcal{M}$ is a quasi-coherent sheaf on $Y$, then by abuse of notation, we denote the push-forward $f_{*} \mathcal{M}$ again by $\mathcal{M}$.

1.2. Acknowledgment. This work was supported by Grant-in-Aid for Young Scientists (20840036) from JSPS.

\section{Noncommutative SChemes}

2.1. Pseudo-schemes. Following [1, page 235], we first define a category.

Definition 2.1. A pseudo-scheme is the pair $(\mathcal{A}, M)$ of a $k$-linear abelian category $\mathcal{A}$ and an object $M \in \mathcal{A}$. A morphism $f:(\mathcal{A}, M) \rightarrow(\mathcal{B}, N)$ of pseudo-schemes is the equivalence class of pairs $\left(f^{*}, \theta\right)$ of a $k$-linear functor $f^{*}: \mathcal{B} \rightarrow \mathcal{A}$ which admits a right adjoint $f_{*}: \mathcal{A} \rightarrow \mathcal{B}$ and an isomorphism $\theta: f^{*} M \cong N$. Here two such pairs $\left(f^{*}, \theta\right)$ and $\left(\left(f^{*}\right)^{\prime}, \theta^{\prime}\right)$ are equivalent if there is an isomorphism $f^{*} \cong\left(f^{*}\right)^{\prime}$ which is compatible with $\theta$ and $\theta^{\prime}$. The composition of morphisms is defined in the obvious way. We denote the category of pseudo-schemes by $\mathcal{P} \mathcal{S}$. A morphism $f$ is said to be flat if its pull-back functor $f^{*}$ is exact.

For a scheme $X$, we denote by $\mathrm{Q} \operatorname{coh}(X)$ the category of quasi-coherent sheaves on $X$. We have a natural functor

$$
\text { (scheme) } \rightarrow \mathcal{P S}, X \mapsto X^{\mathrm{ps}}:=\left(\mathrm{Q} \operatorname{coh}(X), \mathcal{O}_{X}\right) .
$$

From a theorem of Gabriel [5], we can reconstruct $X$ from $X^{\mathrm{ps}}$ (which was generalized to the non-Noetherian case by Rosenberg [12]):

Theorem 2.2 (Reconstruction of schemes). If $X^{\mathrm{ps}} \cong Y^{\mathrm{ps}}$, then $X \cong Y$.

We can also reconstruct morphisms:

Proposition 2.3 (Reconstruction of morphisms). The functor $X \mapsto X^{\mathrm{ps}}$ is faithful. 
Proof. Suppose that $f: Y \rightarrow X=\operatorname{Spec} A$ be a morphism of schemes with $X$ affine. Then $f^{\text {ps }}$ determines a $k$-algebra map $A=\operatorname{End}(A) \rightarrow \Gamma\left(\mathcal{O}_{Y}\right)=\operatorname{End}\left(\mathcal{O}_{Y}\right)$ and so determines $f$.

Next suppose that $f: Y \rightarrow X$ be an arbitrary morphism of schemes. Then applying $f_{*}$ to the structure sheaves of integral closed subschemes of $Y$, we see that $f^{\text {ps }}$ uniquely determines $f$ as the map of sets. For each affine open subset $\iota: U \hookrightarrow X$, applying $f^{*}$ to the sheaves $\iota_{*} \mathcal{M}, \mathcal{M} \in \mathrm{Q} \operatorname{coh}(U)$, we see that $f^{\text {ps }}$ uniquely determines the scheme morphism $\left.f\right|_{f^{-1}(U)}: f^{-1}(U) \rightarrow U$. As a consequence, $f^{\text {ps }}$ uniquely determines $f$. Hence the functor is faithful.

The above results allow us to identify a scheme $X$ (resp. a scheme morphism $f$ ) with $X^{\mathrm{ps}}$ (resp. $f^{\mathrm{ps}}$ ).

\subsection{Noncommutative schemes.}

Definition 2.4. Let $Z$ be a scheme. A finite $N C$ (noncommutative) scheme over $Z$ is the pair $X=(\mathcal{A}, \mathcal{M})$ of a coherent sheaf $\mathcal{A}$ of $\mathcal{O}_{X}$-algebras and a coherent sheaf $\mathcal{M}$ of left $\mathcal{A}$-module. We denote by $\mathrm{Q} \operatorname{coh}(X)=\mathrm{Q} \operatorname{coh}(\mathcal{A})$ the category of quasi-coherent left $\mathcal{A}$-modules and set $X^{\mathrm{ps}}:=(\mathrm{Q} \operatorname{coh}(X), \mathcal{M})$. Like a scheme, we often identify $X$ and $X^{\mathrm{ps}}$.

A morphism $X=(\mathcal{A}, \mathcal{M}) \rightarrow X^{\prime}=\left(\mathcal{A}^{\prime}, \mathcal{M}^{\prime}\right)$ of finite NC schemes over $Z$ is a morphism $X^{\mathrm{ps}} \rightarrow\left(X^{\prime}\right)^{\mathrm{ps}}$ defined by the functor

$$
\mathcal{N} \otimes_{\mathcal{A}^{\prime}}-: \operatorname{Qcoh}\left(X^{\prime}\right) \rightarrow \mathrm{Qcoh}(X)
$$

for some coherent sheaf $\mathcal{N}$ of $\left(\mathcal{A}, \mathcal{A}^{\prime}\right)$-bimodules and an isomorphism $\mathcal{N} \otimes_{\mathcal{A}^{\prime}} \mathcal{M}^{\prime} \cong$ $\mathcal{M}$. Note that the functor has the right adjoint $\mathcal{H o m}_{\mathcal{A}}(\mathcal{N},-)$ and indeed defines a morphism in $\mathcal{P S}$.

We do not construct the correct category of finite $\mathrm{NC}$ schemes over different schemes in this article. Instead we will work in the ambient category $\mathcal{P} \mathcal{S}$.

\section{Alterations and noncommutative Blowups}

Definition 3.1. A morphism $Y \rightarrow X$ of integral schemes is called an alteration (resp. modification) if it is generically finite, dominant and proper (resp. birational and proper). An alteration $Y \rightarrow X$ is said to be normal (resp. regular) if $Y$ is so. A finite-birational factorization of a normal alteration $Y \rightarrow X$ is a factorization of $Y \rightarrow X$ into a finite and dominant morphism $Y \rightarrow \bar{Y}$ and a modification $\bar{Y} \rightarrow X$ with $\bar{Y}$ normal. (This is clearly unique up to isomorphism if exist.) A normal alteration is said to be factorizable if it admits a finite-birational factorization. An alteration $f: Y \rightarrow X$ is said to be Galois if there exists a finite group $G$ of automorphisms of $Y$ such that if we give to $X$ the trivial $G$-action, then $f$ is $G$-equivariant and the field extension $K(Y)^{G} / K(X)$ is purely inseparable.

Lemma 3.2. Let $f: Y \rightarrow X$ be a normal Galois alteration. Suppose that the quotient algebraic space $Y / G$ is a scheme. Then $f$ is factorizable.

Proof. We take $e \in \mathbb{Z}_{\geq 0}$ such that $\left(K(Y)^{G}\right)^{p^{e}} \subset K(X)$. Let $Y / G$ be the quotient variety and $Y / G \rightarrow(Y / G)^{e}$ the morphism corresponding to the inclusion $\mathcal{O}_{Y / G}^{p^{e}} \hookrightarrow$ $\mathcal{O}_{Y / G}$ of sheaves, though this is not a morphism of $k$-schemes unless $k$ is perfect. Let $\bar{Y}$ be the normalization of $(Y / G)^{e}$ in $K(X)$. Then we claim that $f$ factorizes through $\bar{Y}$. To see this, we take affine open coverings $Y=\bigcup \operatorname{Spec} S_{i}$ and $X=\bigcup \operatorname{Spec} R_{i}$ 
such that for each $i, f\left(\operatorname{Spec} S_{i}\right) \subset \operatorname{Spec} R_{i}$ and $\operatorname{Spec} S_{i}$ is stable under the $G$-action. Set $\bar{S}_{i}:=S_{i} \cap K(X)$. Then $\bar{Y}=\bigcup \operatorname{Spec} \bar{S}_{i}$. Since $S_{i} \supset \bar{S}_{i} \supset R_{i}$, the claim holds.

Definition 3.3. For a finite dominant morphism $f: Y \rightarrow X$ of integral schemes, we write $\mathcal{E}_{Y / X}:=\mathcal{E} n d_{\mathcal{O}_{X}}\left(\mathcal{O}_{Y}\right)$. We define the $N C$ blowup associated to $f, \operatorname{NCB}(Y / X)$, to be the finite $\mathrm{NC}$ scheme $\left(\mathcal{E}_{Y / X}, \mathcal{O}_{Y}\right)$ over $X$. Since $\mathcal{O}_{Y}$ is a subring of $\mathcal{E}_{Y / X}$, we have the induced functor $\mathrm{Q} \operatorname{coh}\left(\mathcal{E}_{Y / X}\right) \rightarrow \mathrm{Q} \operatorname{coh}\left(\mathcal{O}_{Y}\right)$, which is identical to $\mathcal{O}_{Y} \mathcal{E}_{Y / X} \otimes_{\mathcal{E}_{Y / X}}-$. We call the corresponding morphism $Y \rightarrow \mathrm{NCB}(Y / X)$ the coforgetful morphism. This is obviously flat. We define the projection $\operatorname{NCB}(Y / X) \rightarrow X$ by $\mathcal{E}_{Y / X} \mathcal{O}_{Y} \otimes_{\mathcal{O}_{X}}-$. The composite morphism

$$
Y \rightarrow \mathrm{NCB}(Y / X) \rightarrow X .
$$

is exactly the original morphism $Y \rightarrow X$.

Definition 3.4. For a factorizable normal alteration $Y \rightarrow X$, if $Y \rightarrow \bar{Y} \rightarrow X$ is the finite-birational factorization, then we define the associated $N C$ blowup, $\operatorname{NCB}(Y / X)$, to be $\operatorname{NCB}(Y / \bar{Y})$.

Remark 3.5. The normality assumption in the above definition is not really necessary, but just for simplicity.

Every factorizable normal alteration $Y \rightarrow X$ factors as $Y \rightarrow \operatorname{NCB}(Y / X) \rightarrow X$.

\section{Frobenius Morphisms}

In this section, we shall define the Frobenius morphism for some class of noncommutative blowups.

From now on, we suppose that the base field $k$ has characteristic $p>0$. We also suppose that every scheme has the finite Frobenius morphism.

\subsection{Equivalent modules.}

Definition 4.1. Let $R$ be a commutative ring. We say that $R$-modules $M$ and $N$ are equivalent if $M$ is isomorphic to a direct summand of $N^{\oplus a}$ for some $a \in \mathbb{Z}_{>0}$ and if $N$ is a direct summand of $M^{\oplus b}$ for some $b \in \mathbb{Z}_{>0}$

Let $X$ be a scheme. We say that coherent sheaves $\mathcal{M}$ and $\mathcal{N}$ on $X$ are equivalent if there exists an affine open covering $X=\bigcup U_{i}$ such that for every $i, \mathcal{M}\left(U_{i}\right)$ and $\mathcal{N}\left(U_{i}\right)$ are equivalent.

Given coherent sheaves $\mathcal{M}$ and $\mathcal{N}$ on a scheme $X$. We think of $\mathcal{M}$ as an $\left(\mathcal{E} n d(\mathcal{M}), \mathcal{O}_{X}\right)$-bimodule and similarly for $\mathcal{N}$. Then $\mathcal{H o m}(\mathcal{M}, \mathcal{N})=\mathcal{H}_{o} m_{\mathcal{O}_{X}}(\mathcal{M}, \mathcal{N})$ is an $(\mathcal{E} n d(\mathcal{N}), \mathcal{E} n d(\mathcal{M}))$-bimodule.

Lemma 4.2. Let $\mathcal{L}, \mathcal{M}$ and $\mathcal{N}$ be coherent sheaves on $X$ which are mutually equivalent.

(1) We have a natural isomorphism of $(\mathcal{E} n d(\mathcal{N}), \mathcal{E} n d(\mathcal{L}))$-bimodules

$$
\mathcal{H o m}(\mathcal{M}, \mathcal{N}) \otimes_{\mathcal{E} n d(\mathcal{M})} \mathcal{H o m}(\mathcal{L}, \mathcal{M}) \cong \mathcal{H o m}(\mathcal{L}, \mathcal{N}) .
$$

In particular

$$
\mathcal{H o m}(\mathcal{M}, \mathcal{N}) \otimes_{\mathcal{E} n d(\mathcal{M})} \mathcal{H o m}(\mathcal{N}, \mathcal{M}) \cong \mathcal{E} n d(\mathcal{N}) .
$$

Hence the functors

$$
\begin{aligned}
& \mathcal{H o m}(\mathcal{M}, \mathcal{N}) \otimes_{\mathcal{E} n d(\mathcal{M})}-: \operatorname{Qcoh}(\mathcal{E} n d(\mathcal{M})) \rightarrow \operatorname{Qcoh}(\mathcal{E} n d(\mathcal{N})) \\
& \mathcal{H o m}(\mathcal{N}, \mathcal{M}) \otimes_{\mathcal{E} n d(\mathcal{N})}-: \operatorname{Qcoh}(\mathcal{E} n d(\mathcal{N})) \rightarrow \operatorname{Qcoh}(\mathcal{E} n d(\mathcal{M}))
\end{aligned}
$$


are equivalences which are inverses to each other.

(2) We have a natural isomorphism of $\left(\mathcal{E} n d(\mathcal{N}), \mathcal{O}_{X}\right)$-bimodules

$$
\mathcal{H} o m(\mathcal{M}, \mathcal{N}) \otimes_{\mathcal{E} n d(\mathcal{M})} \mathcal{M} \cong \mathcal{N} .
$$

Proof. These are well-known to the specialists.

(1) For instance, see [15] for the affine case. The general case easily follows.

(2) Choose a splitting injection $\mathcal{N} \rightarrow \mathcal{M}^{\oplus r}$. Then we have the commutative diagram of right $\mathcal{O}_{X}$-modules

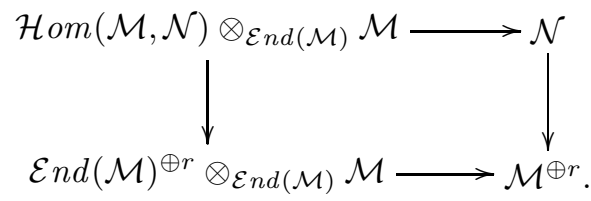

Here the vertical arrows are splitting injections and the bottom arrow is an isomorphism. Now we easily see that the top arrow is also an isomorphism.

4.2. F-steady morphisms and Frobenius morphisms. For a scheme $X$, we write the $e$-iterated $k$-linear Frobenius as

$$
F^{e}=F_{X}^{e}: X_{e} \rightarrow X .
$$

Sometimes we simply call this the $e$-th Frobenius of $X$. A key observation is that the morphism $F^{e}$ factors as $X_{e} \rightarrow \operatorname{NCB}\left(X_{e} / X\right) \rightarrow X$ (see Definition 3.3).

Definition 4.3. Let $f: Y \rightarrow X$ be a finite dominant morphism of integral normal schemes. We say that $f$ is $F$-steady if for every $e, \mathcal{O}_{Y}$ and $\mathcal{O}_{Y_{e}}$ are equivalent $\mathcal{O}_{X}$-modules.

Example 4.4. If $Y$ is regular, then $f$ is F-steady. Indeed being flat over $\mathcal{O}_{Y}$, $\mathcal{O}_{Y_{e}}$ is locally isomorphic to $\mathcal{O}_{Y}^{\oplus r}, r>0$, as an $\mathcal{O}_{Y}$-module and hence also as an $\mathcal{O}_{X}$-module.

From Lemma4.2 for an $F$-steady morphism $f: Y \rightarrow X$, we have an isomorphism

$$
\operatorname{NCB}\left(Y_{e} / X\right) \cong \operatorname{NCB}\left(Y_{e^{\prime}} / X\right), e, e^{\prime} \geq 0 \text {. }
$$

We also define a morphism

$$
\operatorname{NCB}\left(Y_{e} / X_{e}\right) \rightarrow \operatorname{NCB}\left(Y_{e} / X\right),
$$

which we call the coforgetful morphism, as follows. We think of $\mathcal{O}_{Y}$ as a subring of $\mathcal{O}_{Y_{e}}$ in the obvious way. Then $\mathcal{E}_{Y_{e} / X_{e}}$ is a subring of $\mathcal{E}_{Y_{e} / X}$. Hence we have a natural morphism $\operatorname{NCB}\left(Y_{e} / X_{e}\right) \rightarrow \operatorname{NCB}\left(Y_{e} / X\right)$ defined by $\mathcal{E}_{Y_{e} / X_{e}} \mathcal{E}_{Y_{e} / X} \otimes_{\mathcal{E}_{Y_{e} / X}}-$.

Definition 4.5. Let $f: Y \rightarrow X$ be an F-steady morphism. We define the $e$-th Frobenius of $\mathrm{NCB}(Y / X)$ to be the composite

$$
F^{e}=F_{\mathrm{NCB}(Y / X)}^{e}: \operatorname{NCB}\left(Y_{e} / X_{e}\right) \stackrel{\text { cofor. }}{\longrightarrow} \mathrm{NCB}\left(Y_{e} / X\right) \stackrel{\sim}{\longrightarrow} \mathrm{NCB}(Y / X) .
$$

By construction, this is flat, which we call the Kunz regularity of $\operatorname{NCB}(Y / X)$. (Recall that from Kunz [11, a scheme is regular if and only if its Frobenius morphisms are flat.) The morphism is also directly defined by the functor

$$
\mathcal{E}_{Y_{e} / X_{e}} \mathcal{H o m _ { \mathcal { O } _ { X } }}\left(\mathcal{O}_{Y}, \mathcal{O}_{Y_{e}}\right) \otimes_{\mathcal{E}_{Y / X}}-
$$


Corollary 4.6. Suppose that $k$ is algebraically closed. Then for any variety $X$ (that is, an integral scheme of finite type over $k$ ), there exists an $N C$ blowup $\operatorname{NCB}(Y / X)$ which is Kunz regular.

Proof. From de Jong's theorem [4, 7.3], there exists a regular Galois alteration $Y \rightarrow X$. Let $Y \rightarrow \bar{Y} \rightarrow X$ be the finite-birational factorization. Since $Y$ is regular, $Y \rightarrow \bar{Y}$ is F-steady. Hence $\operatorname{NCB}(Y / \bar{Y})=\operatorname{NCB}(Y / X)$ is Kunz regular.

Remark 4.7. Bondal and Orlov 2 conjectured the following: Let $Y \rightarrow X$ be a finite morphism of varieties such that $X$ has canonical singularities and $Y$ is regular. Then the derived category of $\mathcal{E}_{Y / X}$-modules is a minimal categorical desingularization. Their conjecture and the above corollary seem somehow related.

4.3. Compatibilities of Frobenius morphisms. In this subsection, to justify our definition of the Frobenius morphism, we show some compatibilities of it (see also Section (6). We suppose that $f: Y \rightarrow X$ is an F-steady finite dominant morphism of integral normal schemes.

Proposition 4.8. The diagram

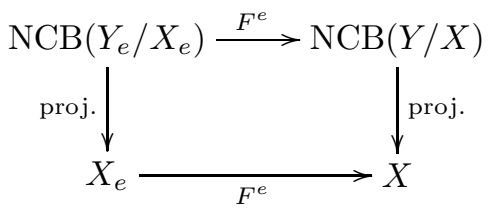

is commutative.

Proof. From Lemma 4.2, we have isomorphisms of $\left(\mathcal{E}_{Y_{e} / X_{e}}, \mathcal{O}_{X}\right)$-bimodules

$$
\mathcal{O}_{Y_{e}} \otimes_{\mathcal{O}_{X_{e}}} \mathcal{O}_{X_{e}} \cong \mathcal{O}_{Y_{e}} \cong \mathcal{H} o m_{\mathcal{O}_{X}}\left(\mathcal{O}_{Y}, \mathcal{O}_{Y_{e}}\right) \otimes_{\mathcal{E}_{Y / X}} \mathcal{O}_{Y}
$$

which proves the proposition.

Proposition 4.9. Suppose that $Y$ is regular. Then the diagram

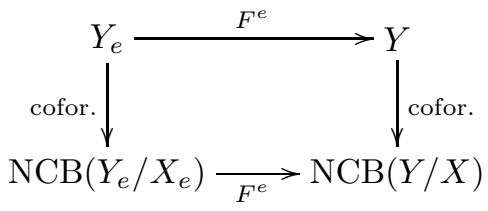

is commutative.

Proof. Because $\mathcal{O}_{Y_{e}}$ is a locally free $\mathcal{O}_{Y}$-module, the canonical map

$$
\mathcal{O}_{Y_{e}} \otimes_{\mathcal{O}_{Y}} \mathcal{E}_{Y / X} \rightarrow \mathcal{H o m} \operatorname{O}_{X}\left(\mathcal{O}_{Y}, \mathcal{O}_{Y_{e}}\right) \text {. }
$$

is an isomorphism, which proves the proposition.

Lemma 4.10. For $e^{\prime} \geq e \geq 0$, the diagram

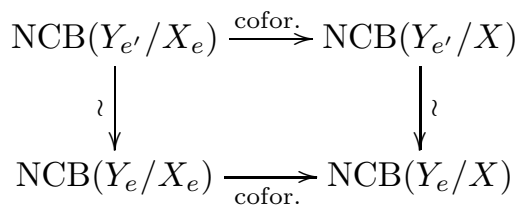

is commutative. 
Proof. There exists a natural morphism

$$
\begin{aligned}
& \alpha: \mathcal{H o m}_{\mathcal{O}_{X_{e}}}\left(\mathcal{O}_{Y_{e}}, \mathcal{O}_{Y_{e^{\prime}}}\right) \otimes_{\mathcal{E}_{Y_{e} / X_{e}}} \mathcal{E}_{Y_{e} / X} \rightarrow \mathcal{H o m}{\mathcal{O}_{X}}\left(\mathcal{O}_{Y_{e}}, \mathcal{O}_{Y_{e^{\prime}}}\right) \\
& \phi \otimes \psi \mapsto \phi \circ \psi
\end{aligned}
$$

We claim that this is an isomorphism, which proves the lemma.

Let $U \subset X$ be an open subset such that $X \backslash U$ has codimension $\geq 2$ and $f^{-1}(U) \subset Y$ is regular. Then locally on $U$, we have an isomorphism of $\mathcal{O}_{X_{e}}$ modules $\mathcal{O}_{Y_{e^{\prime}}} \cong \mathcal{O}_{Y_{e}}^{\oplus r}$ for some $r$. Hence locally on $U$, the source and target of $\alpha$ are both isomorphic to $\mathcal{E}_{Y_{e} / X}^{\oplus r}$. It is now easy to see that $\alpha$ is an isomorphism over $U$.

Moreover both hand sides are flat right $\mathcal{E}_{Y_{e} / X \text {-modules and hence locally iso- }}$ morphic to direct summands of $\mathcal{E}_{Y_{e} / X}^{\oplus l}$ for some $l$. From the normality assumption, $\mathcal{E}_{Y_{e} / X}^{\oplus l}$ is a reflexive $\mathcal{O}_{X}$-module (see [7, Prop. 1.6]) and so are its direct summands. So $\alpha$ is an isomorphism all over $X$. We have proved the claim and the lemma.

Corollary 4.11. For $e, e^{\prime} \geq 0$, the diagram

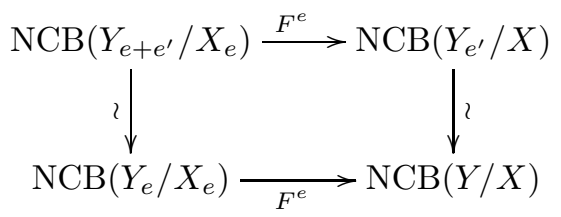

is commutative.

Proof. If $e^{\prime} \geq e$, then from Lemmas 4.2 and 4.10, the diagram

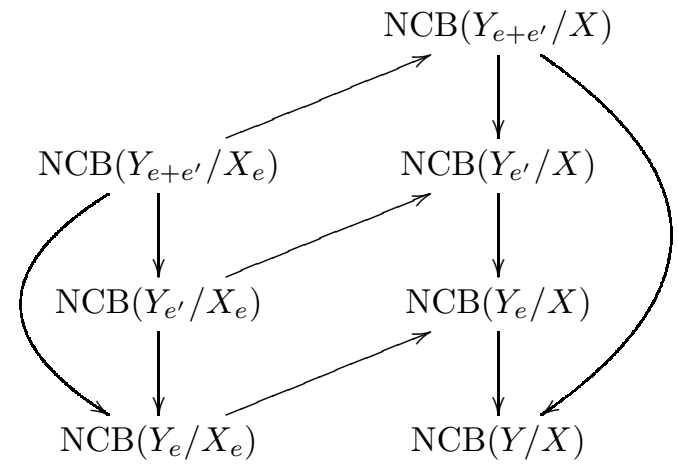

is commutative. Now the corollary follows from our definition of the Frobenius morphism. 
If $e^{\prime}<e$, then similarly the diagram

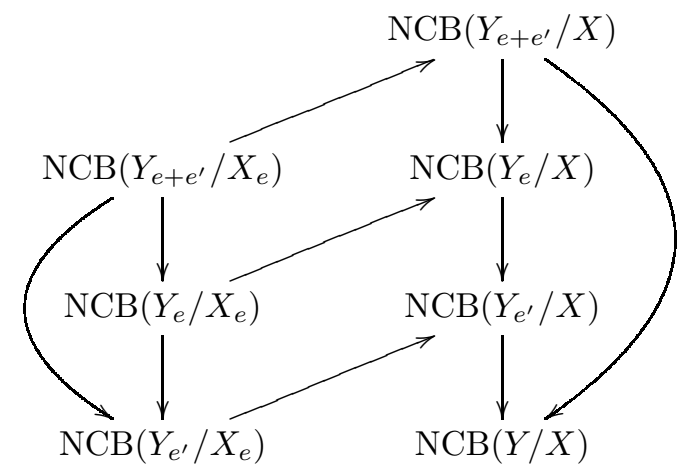

is commutative and the corollary follows.

Corollary 4.12. For $e, e^{\prime} \geq 0$, the diagram

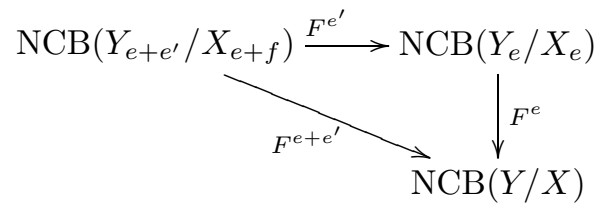

is commutative. Namely we have $F^{e+e^{\prime}}=F^{e} \circ F^{e}$. In particular, the e-th Frobenius of $\operatorname{NCB}(Y / X)$ is the e-iterate of the first Frobenius.

Proof. This follows from the commutativity of the diagram

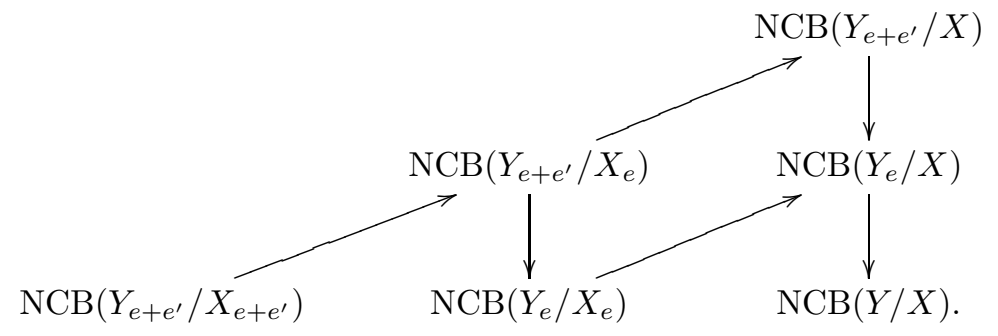

\section{D-BLOWups}

Among NC blowups, especially interesting are the ones associated to Frobenius morphisms of schemes.

Definition 5.1. For an integral scheme $X$, we define the $e$-th $D$-blowup of $X$ as $\operatorname{DB}_{e}(X):=\operatorname{NCB}\left(X_{e} / X\right)$.

Remark 5.2. The $D$-blowup can be regarded as the noncommutative counterpart of the F-blowup (see [15]).

Definition 5.3 (Hochster-Roberts [8]). Let $X=\operatorname{Spec} R$ be an integral scheme. We say that $R$ and $X$ are $F$-pure if $R \hookrightarrow R_{e}$ splits as an $R$-module map. 
Definition 5.4 (Smith-Van den Bergh [13). Suppose that $R$ is a complete local Noetherian domain so that the Krull-Schmidt decomposition holds for finitely generated $R$-modules. Then $R$ and Spec $R$ are said to be FFRT (finite $F$-representation type) if there are finitely many indecomposable $R$-modules $M_{i}, i=1, \ldots, n$, such that for any $e, R_{e}$ is isomorphic to $\bigoplus_{i=1}^{n} M_{i}^{\oplus r_{i}}, r_{i} \geq 0$, as an $R$-module.

Proposition 5.5. Let $R$ be a complete local Noetherian normal domain. Suppose that $X=\operatorname{Spec} R$ is F-pure and FFRT. Then for sufficiently large e, the Frobenius morphism $F_{X}^{e}: X_{e} \rightarrow X$ is F-steady.

Proof. Let $M_{i}, i=1, \ldots, n$, be the irredundant set of indecomposable modules as in the above definition. Then there exists $e_{0}$ such that for every $e \geq e_{0}, R_{e}$ is isomorphic, as an $R$-module, to $\bigoplus_{i=1}^{n} M_{i}^{\oplus r_{i}}, r_{i}>0$. Hence $X_{e} \rightarrow X$ is F-steady.

As a corollary, we obtain the following.

Corollary 5.6. Let $X$ be an integral normal scheme with F-pure and FFRT singularities. Namely the completion of every local ring of $X$ is F-pure and FFRT. Then for sufficiently large $e, \mathrm{DB}_{e}(X)$ is Kunz regular.

Example 5.7. Normal toric singularities and tame quotient singularities are Fpure and FFRT. See [14] for other examples.

\section{Comparing Frobenius morphisms of commutative and NONCOMMUTATIVE BLOWUPS}

Let $X=\operatorname{Spec} R$ and $Y=\operatorname{Spec} S$ be integral normal affine schemes and $f: Y \rightarrow$ $X$ an $F$-steady finite dominant morphism. Let $g: Z \rightarrow X$ be a modification which is a flattening of $f$, that is, $\mathcal{S}:=g^{*} \mathcal{O}_{Y} /$ tors is locally free.

Lemma 6.1. We have $\Gamma(\mathcal{S})=S$.

Proof. Since $\mathcal{O}_{Y}$ is a torsion-free and normal $\mathcal{O}_{X}$-module, it is a reflexive $\mathcal{O}_{X^{-}}$ module (see [7, Prop. 1.6]). Hence there exists an open subset $U \subset X$ such that $X \backslash U$ has codimension $\geq 2$ and $\mathcal{O}_{Y}$ is locally free on $U$. Since $X$ is normal, from Zariski's main theorem, $\left.\left(g_{*} \mathcal{S}\right)\right|_{U}=g_{*} g^{*}\left(\left.\mathcal{O}_{Y}\right|_{U}\right)=\left.\mathcal{O}_{Y}\right|_{U}$. It follows that the natural morphism $\mathcal{O}_{Y} \rightarrow g_{*} \mathcal{S}$ is an injection into a torsion-free sheaf which is an isomorphism over $U$. Since $\mathcal{O}_{Y}$ is reflexive, this is an isomorphism. Therefore we have

$$
\Gamma(\mathcal{S})=\Gamma\left(g_{*} \mathcal{S}\right)=\Gamma\left(\mathcal{O}_{Y}\right)=S .
$$

Set $W:=\operatorname{Spec} \mathcal{S}, E:=\operatorname{End}_{R}(S)$ and $\mathcal{E}:=\mathcal{E} n d_{\mathcal{O}_{Z}}(\mathcal{S})$. Then from the preceding lemma, $E=\Gamma(\mathcal{E})$. Since $\mathcal{S}$ is locally free, the projection

$$
h: \mathrm{NCB}(W / Z) \rightarrow Z,
$$

which is defined by $\mathcal{S} \otimes_{\mathcal{O}_{Z}}-$, is an isomorphism.

For $\mathcal{M} \in \mathrm{Q} \operatorname{coh}(\mathcal{E})=\mathrm{Q} \operatorname{coh}(\operatorname{NCB}(W / Z)), \Gamma(\mathcal{M})$ is a left $A$-module, that is, $\mathcal{M} \in \mathrm{Q} \operatorname{coh}(\mathrm{NCB}(Y / X))$. We define a left exact functor

$$
\Phi: \mathrm{Q} \operatorname{coh}(Z) \rightarrow \mathrm{Q} \operatorname{coh}(\mathrm{NCB}(Y / X)), \mathcal{M} \mapsto \Gamma\left(h^{*} \mathcal{M}\right) .
$$

Identifying $X, Y, Z$ with $X_{e}, Y_{e}, Z_{e}$ respectively, we also define

$$
\Phi_{e}: \operatorname{Qcoh}\left(Z_{e}\right) \rightarrow \operatorname{Qcoh}\left(\mathrm{NCB}\left(Y_{e} / X_{e}\right)\right) .
$$


Proposition 6.2. The diagram

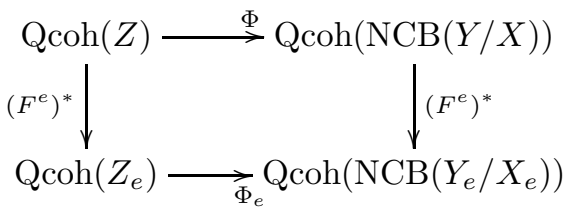

is commutative up to isomorphism of functors.

Proof. We claim that for $\mathcal{M} \in \mathrm{Q} \operatorname{coh}(\mathcal{E})$, there exists a natural isomorphism

$$
\operatorname{Hom}_{R}\left(S, S_{e}\right) \otimes_{E} \Gamma(\mathcal{M}) \cong \Gamma\left(\mathcal{H o m} \mathcal{O}_{Z}\left(\mathcal{S}, \mathcal{S}_{e}\right) \otimes_{\mathcal{E}} \mathcal{M}\right) .
$$

Obviously there exists a natural morphism from the left-hand side to the right-hand side. Since the claim is local on $X$, to show this, we may suppose that $S$ and $S_{e}$ are equivalent $R$-modules. By definition, for some $r, S_{e}$ is isomorphic to a direct summand of $S^{\oplus r}$. It follows that $\operatorname{Hom}_{R}\left(S, S_{e}\right)$ is isomorphic to a direct summand of $E^{\oplus r}$ and $\mathcal{H o m}_{\mathcal{O}_{Z}}\left(\mathcal{S}, \mathcal{S}_{e}\right)$ is isomorphic to a direct summand of $\mathcal{E}^{\oplus r}$. Now the claim is easy to see.

We have natural isomorphisms

$$
\begin{aligned}
& \left(\left(F^{e}\right)^{*} \circ \Phi\right)(\mathcal{M}) \\
& =\operatorname{Hom}_{R}\left(S, S_{e}\right) \otimes_{E} \Gamma\left(\mathcal{S} \otimes_{\mathcal{O}_{Z}} \mathcal{M}\right) \\
& \cong \Gamma\left(\mathcal{H} o m_{\mathcal{O}_{Z}}\left(\mathcal{S}, \mathcal{S}_{e}\right) \otimes_{\mathcal{E}} \mathcal{S} \otimes_{\mathcal{O}_{Z}} \mathcal{M}\right) \\
& \cong \Gamma\left(\mathcal{S}_{e} \otimes_{\mathcal{O}_{Z}} \mathcal{M}\right) \\
& \cong \Gamma\left(\mathcal{S}_{e} \otimes_{\mathcal{O}_{Z_{e}}} \mathcal{O}_{Z_{e}} \otimes_{\mathcal{O}_{Z}} \mathcal{M}\right) \\
& \cong \Gamma\left(\mathcal{S}_{e} \otimes_{\mathcal{O}_{Z_{e}}}\left(F^{e}\right)^{*} \mathcal{M}\right) \\
& \cong\left(\Phi_{e} \circ\left(F^{e}\right)^{*}\right)(\mathcal{M}) .
\end{aligned}
$$

Thus the proposition holds.

We have the right derived functor of $\Phi$

$$
\mathbf{R} \Phi: D^{+}(\mathrm{Q} \operatorname{coh}(Z)) \rightarrow D^{+}(\mathrm{Q} \operatorname{coh}(\mathrm{NCB}(Y / X))) .
$$

Similarly for $\Phi_{e}$.

Corollary 6.3. The diagram

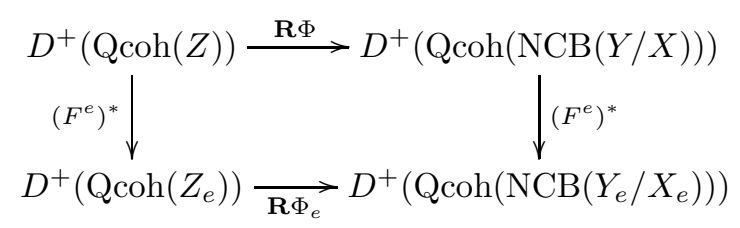

is commutative up to isomorphism of functors.

Proof. We have

$$
\left(F^{e}\right)^{*} \circ \mathbf{R} \Phi \cong \mathbf{R}\left(\left(F^{e}\right)^{*} \circ \Phi\right) \cong \mathbf{R}\left(\Phi \circ\left(F^{e}\right)^{*}\right) \cong(\mathbf{R} \Phi) \circ\left(F^{e}\right)^{*} .
$$


The functor $\mathbf{R} \Phi$ maps $D^{b}(\operatorname{Coh}(Z))$ into $D^{b}(\operatorname{Coh}(\operatorname{NCB}(Y / X)))$. Here $\operatorname{Coh}(?) \subset$ Qcoh(?) denotes the full subcategory of coherent sheaves. As shown in [6, 10, 3, 16, in some situations, the functor

$$
\mathbf{R} \Phi: D^{b}(\mathrm{Coh}(Z)) \rightarrow D^{b}(\operatorname{Coh}(\mathrm{NCB}(Y / X)))
$$

is an equivalence, a kind of Fourier-Mukai transform. Then through this equivalence, the Frobenius morphisms on both hand sides correspond to each other at the level of derived category.

Example 6.4. Let $G \subset S L_{d}(k)$ be a small finite subgroup of order prime to $p$ with $d=2,3$. Set $R:=k\left[x_{1}, \ldots, x_{d}\right]^{G}$ and $X:=\operatorname{Spec} R$. Let $Y$ be either $\mathbb{A}_{k}^{d}$ or $X_{e}$ for $e \gg 0$, and let $Z$ be the universal flattening of $Y \rightarrow X$, which is isomorphic to the $G$-Hilbert scheme of Ito-Nakamura [9] (for the case $Y=X_{e}$, see [15, 17]). Then the above functor is an equivalence (for instance, see [15, 16]).

\section{REFERENCES}

[1] M. Artin and J. J. Zhang. Noncommutative projective schemes. Adv. Math., 109(2):228-287, 1994.

[2] A. Bondal and D. Orlov. Derived categories of coherent sheaves. In Proceedings of the International Congress of Mathematicians, Vol. II (Beijing, 2002), pages 47-56, Beijing, 2002. Higher Ed. Press.

[3] Tom Bridgeland, Alastair King, and Miles Reid. The McKay correspondence as an equivalence of derived categories. J. Amer. Math. Soc., 14(3):535-554 (electronic), 2001.

[4] A. J. de Jong. Smoothness, semi-stability and alterations. Inst. Hautes Études Sci. Publ. Math., (83):51-93, 1996.

[5] Pierre Gabriel. Des catégories abéliennes. Bull. Soc. Math. France, 90:323-448, 1962.

[6] G. Gonzalez-Sprinberg and J.-L. Verdier. Construction géométrique de la correspondance de McKay. Ann. Sci. École Norm. Sup. (4), 16(3):409-449 (1984), 1983.

[7] Robin Hartshorne. Stable reflexive sheaves. Math. Ann., 254(2):121-176, 1980.

[8] Melvin Hochster and Joel L. Roberts. The purity of the Frobenius and local cohomology. Advances in Math., 21(2):117-172, 1976.

[9] Yukari Ito and Iku Nakamura. McKay correspondence and Hilbert schemes. Proc. Japan Acad. Ser. A Math. Sci., 72(7):135-138, 1996.

[10] M. Kapranov and E. Vasserot. Kleinian singularities, derived categories and Hall algebras. Math. Ann., 316(3):565-576, 2000.

[11] Ernst Kunz. Characterizations of regular local rings for characteristic p. Amer. J. Math., 91:772-784, 1969.

[12] Alexander L. Rosenberg. Noncommutative schemes. Compositio Math., 112(1):93-125, 1998.

[13] Karen E. Smith and Michel Van den Bergh. Simplicity of rings of differential operators in prime characteristic. Proc. London Math. Soc. (3), 75(1):32-62, 1997.

[14] Shunsuke Takagi and Ryo Takahashi. $D$-modules over rings with finite $F$-representation type. Math. Res. Lett., 15(3):563-581, 2008.

[15] Yukinobu Toda and Takehiko Yasuda. Noncommutative resolution, F-blowups and Dmodules. arXiv:0810.1804, to appear in Adv. Math.

[16] Michel Van den Bergh. Non-commutative crepant resolutions. In The legacy of Niels Henrik Abel, pages 749-770. Springer, Berlin, 2004.

[17] Takehiko Yasuda. Universal flattening of Frobenius. arXiv:0706.2700v3.

Department of Mathematics and Computer Science, Kagoshima University, 1-21-35 Korimoto, Kagoshima 890-0065, JAPAN

E-mail address: yasuda@sci.kagoshima-u.ac.jp 